\title{
LA-UR-19-26114
}

Approved for public release; distribution is unlimited.

Title: Design-by-contract and test-driven software development

Author(s): $\quad$ Hakel, Peter

Intended for: guest lecture at the University of Colorado Boulder

Issued: $\quad$ 2019-07-29 (rev.1) 
Disclaimer:

Los Alamos National Laboratory, an affirmative action/equal opportunity employer, is operated by Triad National Security, LLC for the National Nuclear Security Administration of U.S. Department of Energy under contract 89233218CNA000001. By approving this article, the publisher recognizes that the U.S. Government retains nonexclusive, royalty-free license to publish or reproduce the published form of this contribution, or to allow others to do so, for U.S. Government purposes. Los Alamos National Laboratory requests that the publisher identify this article as work performed under the auspices of the U.S. Department of Energy. Los Alamos National Laboratory strongly supports academic freedom and a researcher's right to publish; as an institution, however, the Laboratory does not endorse the viewpoint of a publication or guarantee its technical correctness. 


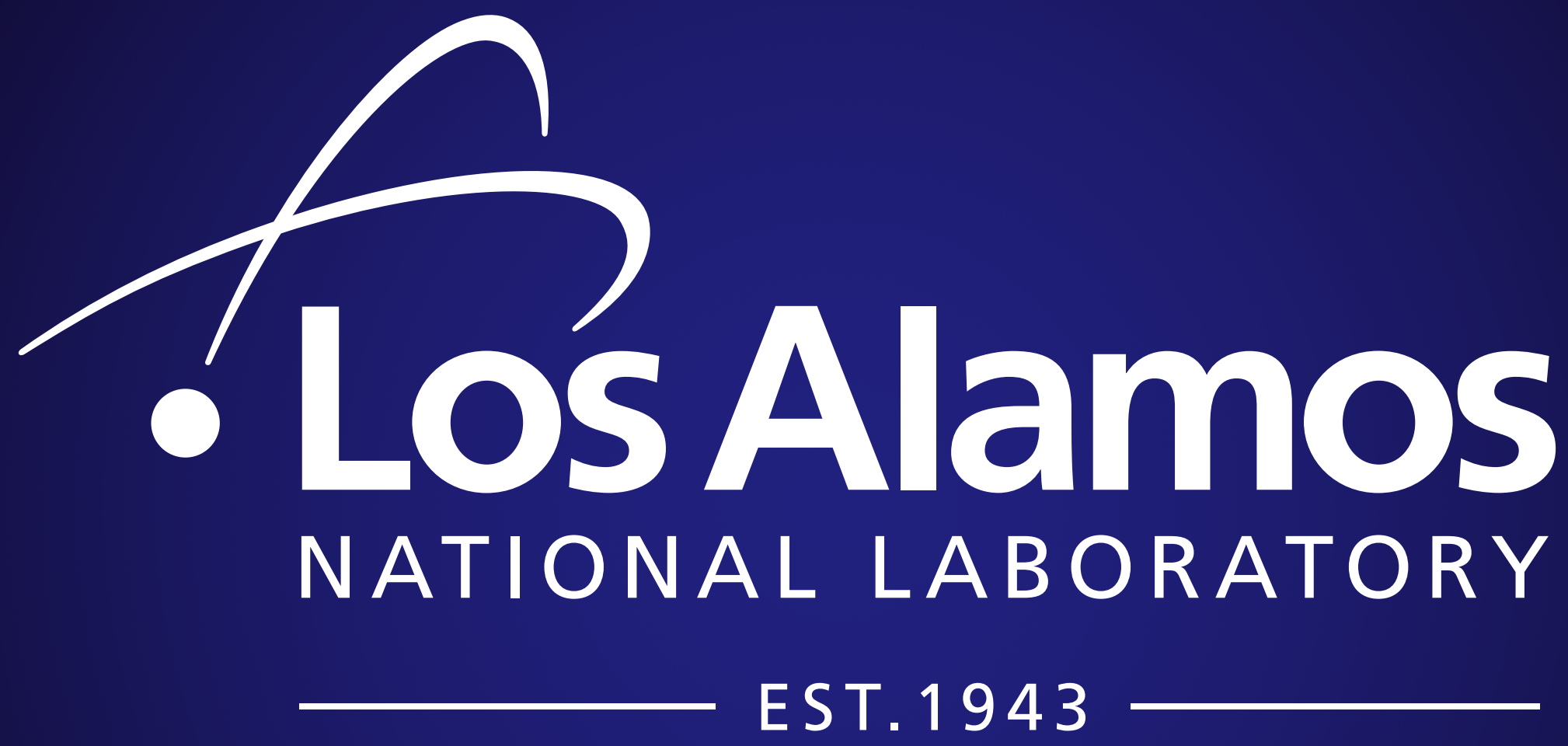




\section{Design-by-contract and test-driven software development}

Guest lecture at the Computational Multi-Physics Production Software Development class University of Colorado Boulder

Peter Hakel

22 July 2019 


\section{Outline}

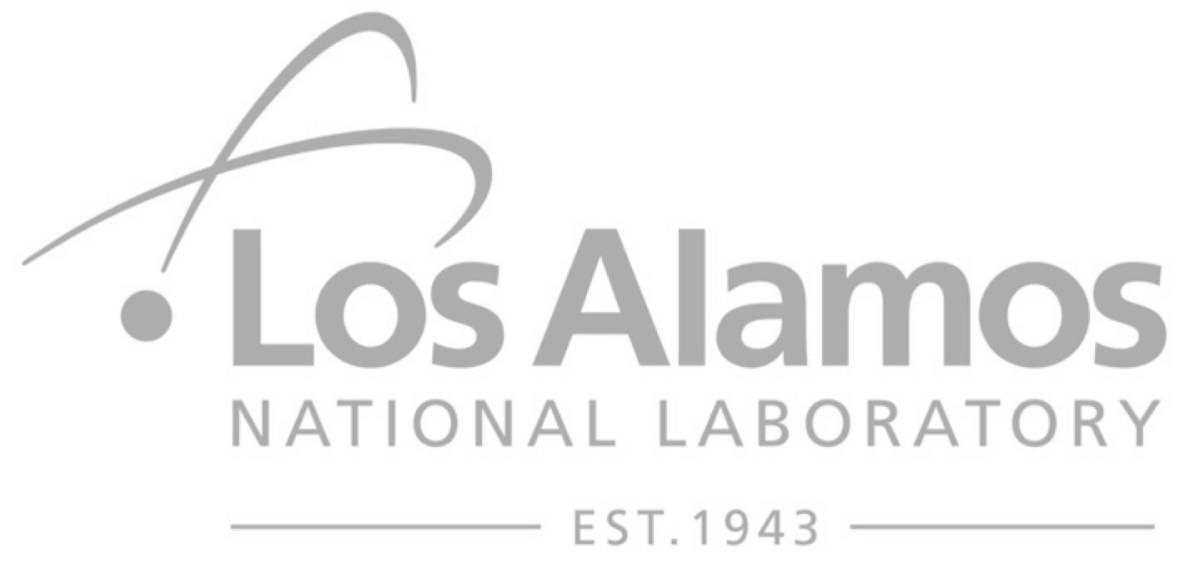

- Introduction

- Documentation

- Contracts

- Unit testing

- Compilation

- Examples

- Summary 


\section{Spectroscopy in visible and infrared regimes}

\section{CompB:}

$\mathrm{T}=1574 \mathrm{~K}$ (Planckian source)

$\rho=0.5 \mathrm{~g} / \mathrm{cm}^{3}$

thin shell, radius $15 \mathrm{~cm}$

spherical geometry

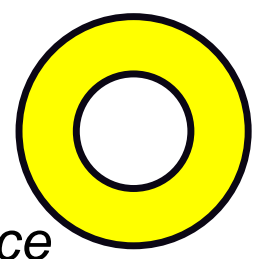

Source

("fireball")

EOS and opacity data

were provided by

Eddy Timmermans (T-4)

Josh Coe (T-1)

Leanne Duffy (AOT-AE),

Cristiano Nisoli (T-4).

Dima Mozyrsky (T-4).

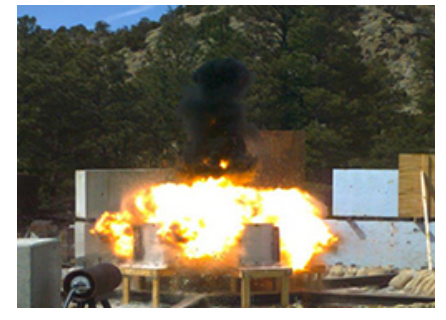

Transport medium (air)
Air:

$\mathrm{T}=296 \mathrm{~K}$ standard conditions length $100 \mathrm{~m}$ planar geometry
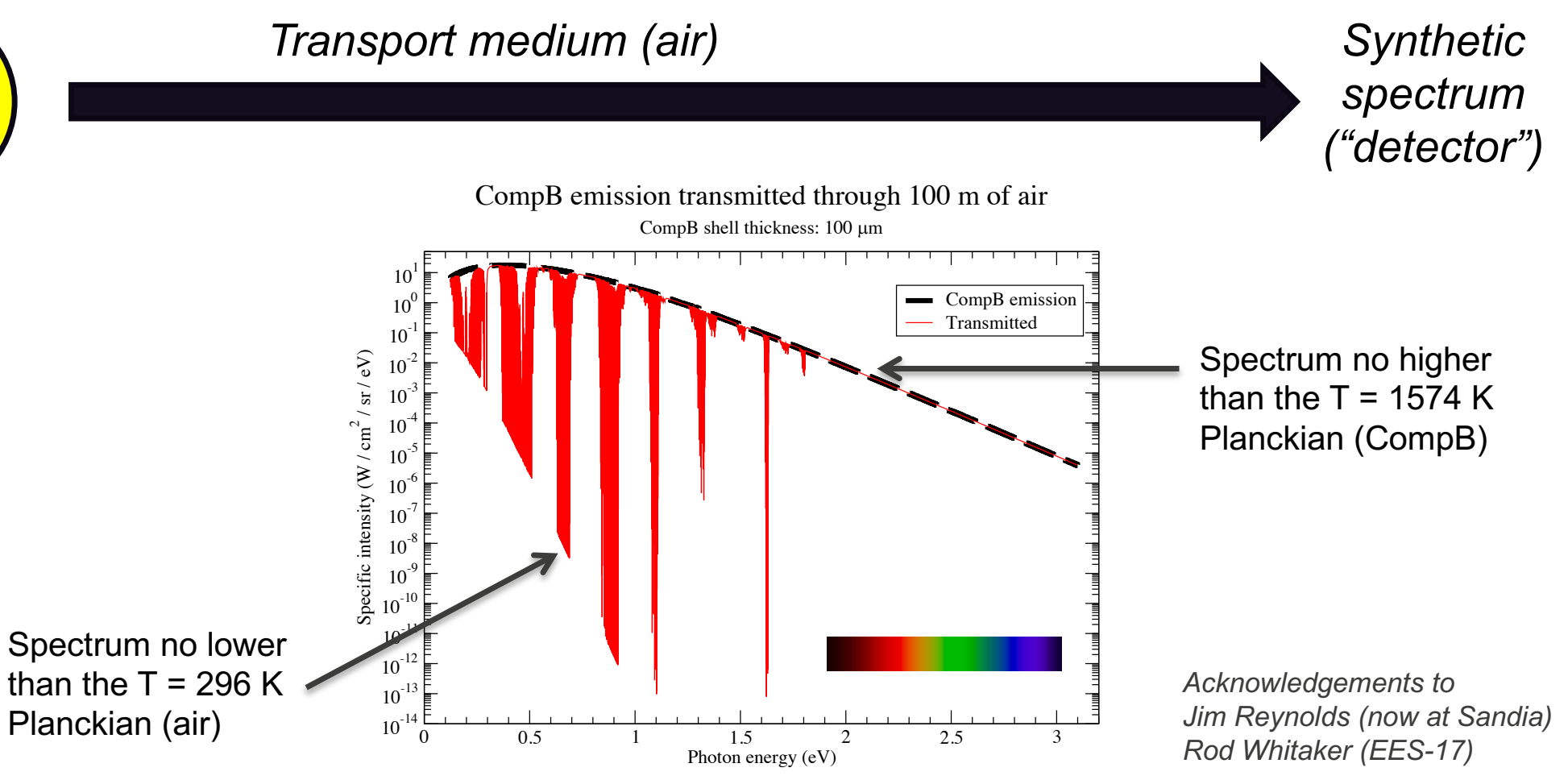


\section{Spectroscopy in $\mathrm{x}$-ray regime}

P. Hakel et al., "X-ray spectroscopic diagnostics and modeling of polar-drive implosion experiments on the National Ignition Facility", Physics of Plasmas 21, 063306 (2014).

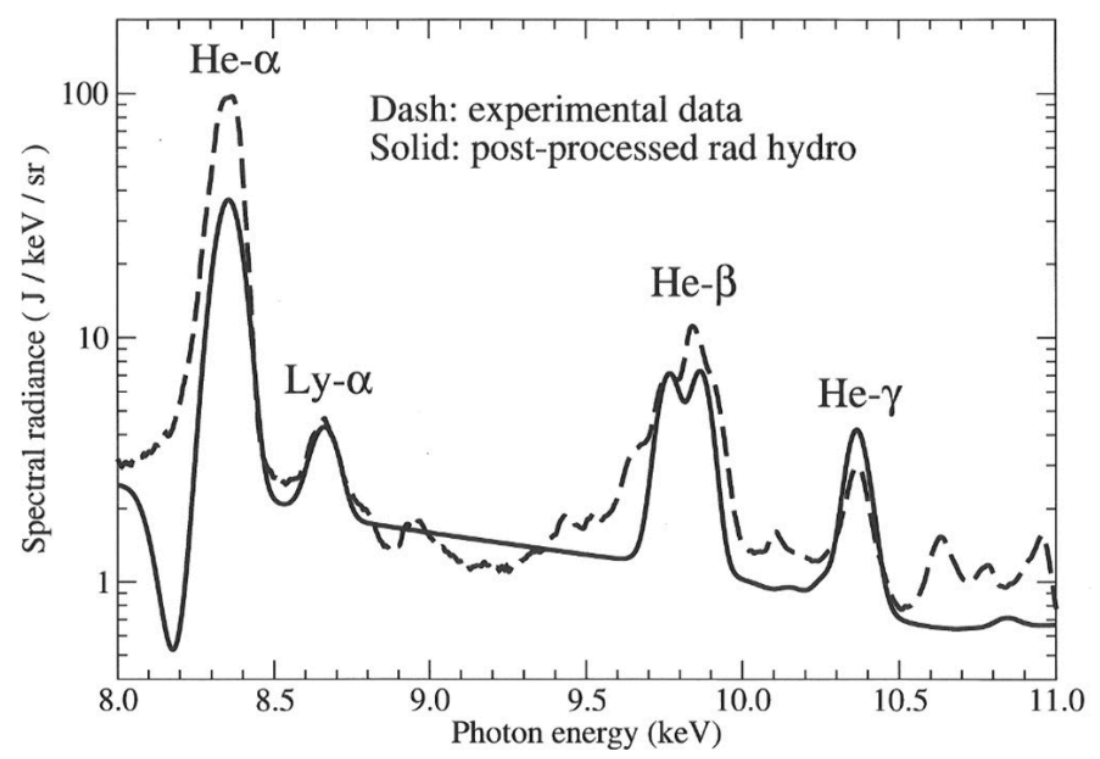

FIG. 9. Comparison of instrumentally broadened synthetic spectrum from post-processed rad hydro simulation with Supersnout II experimental data for the N130617 target (Cu dopant).
NIF target bay Image credit:

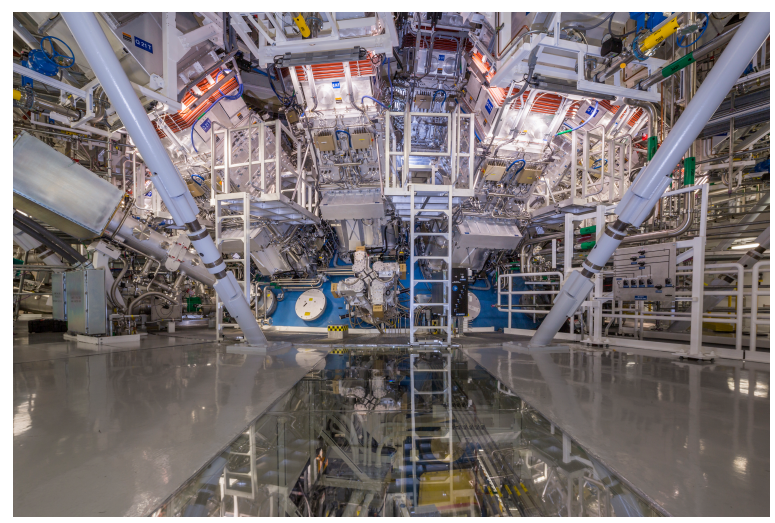

Lawrence Livermore National Laboratory

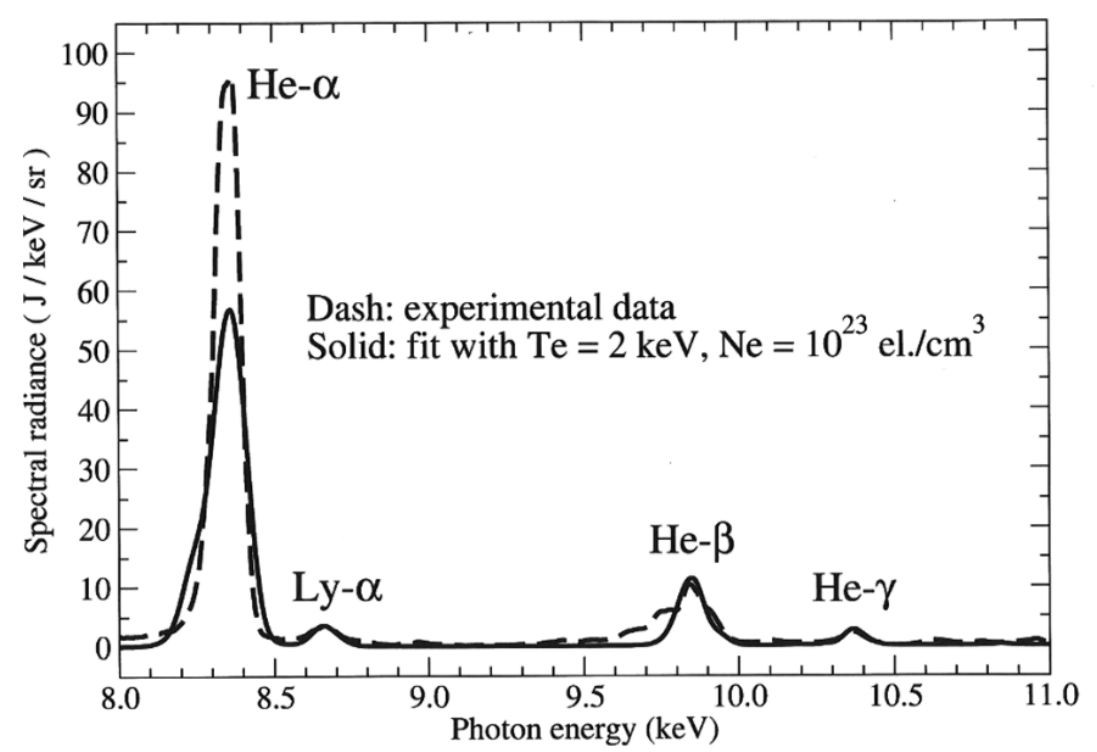

FIG. 11. Comparison of instrumentally broadened synthetic spectrum from a single temperature-density fit with continuum-subtracted Supersnout II experimental data for the N130617 target (Cu dopant). 


\section{FESTR: Finite-Element Spectral Transfer of Radiation}

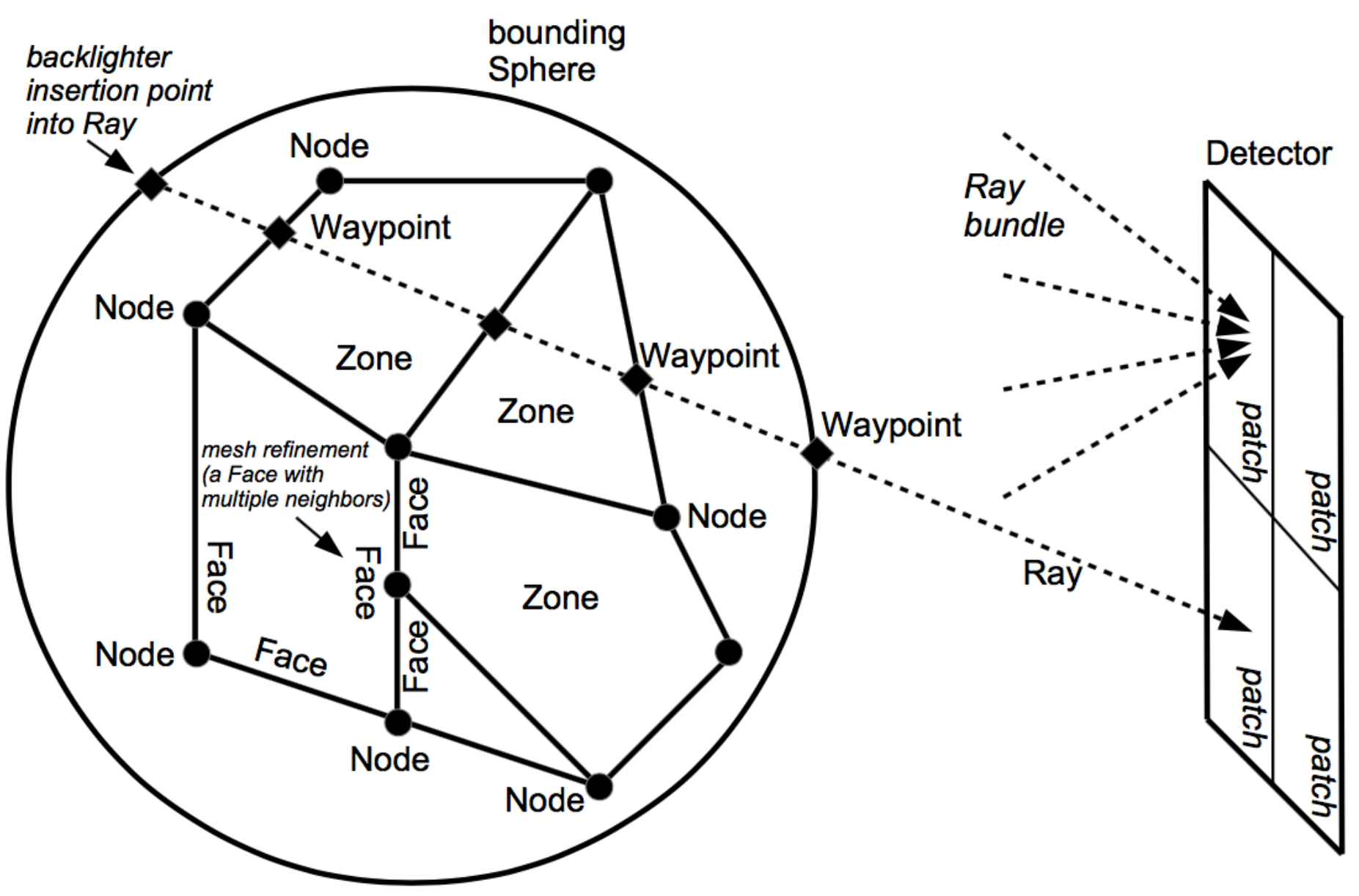

P. Hakel, Computer Physics Communications 206, 415 (2016).

https://github.com/LANLhakel/FESTR 


\section{Doxygen documentation}

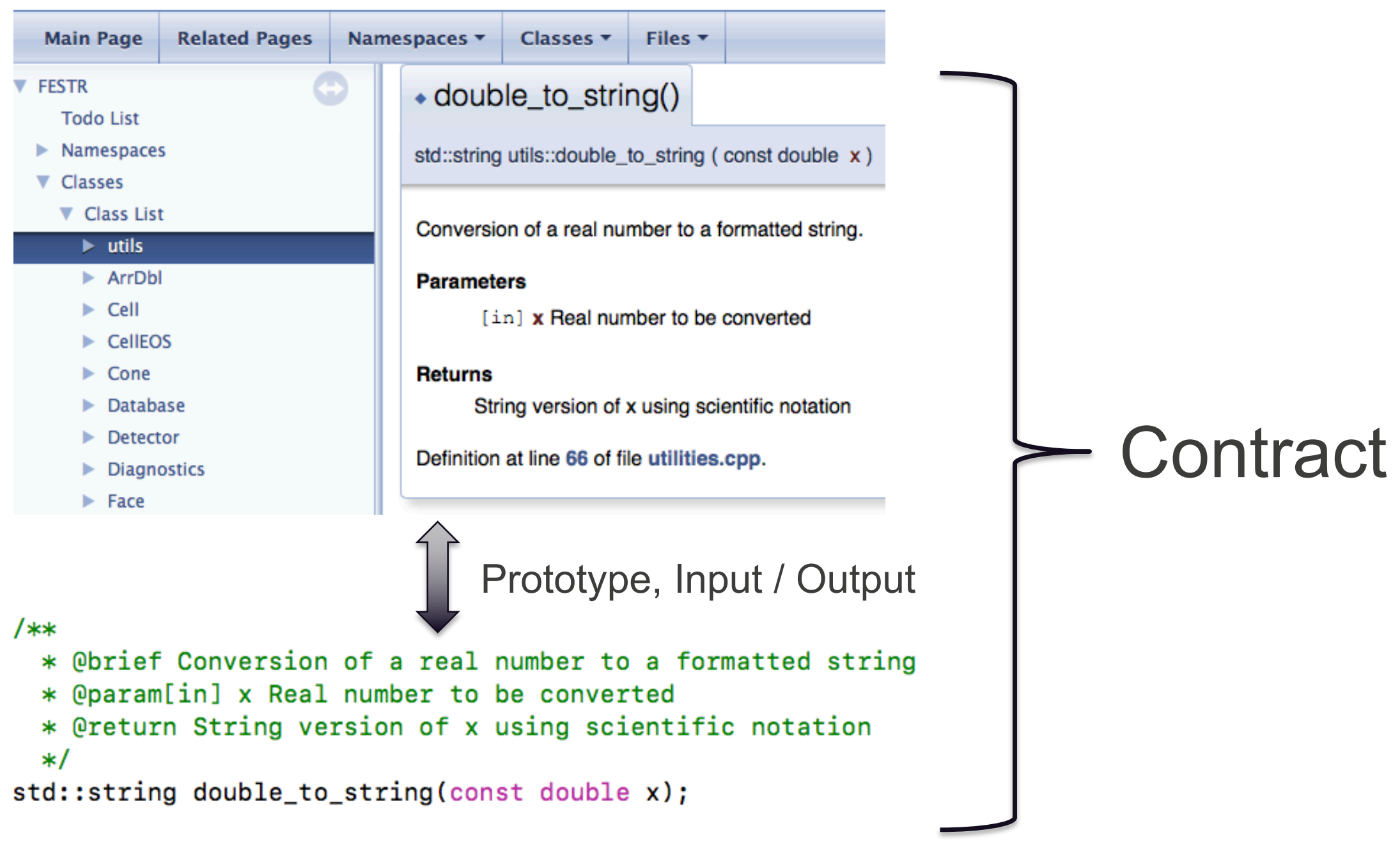




\section{Example of a unit test}

\{

utilities

name

category

s

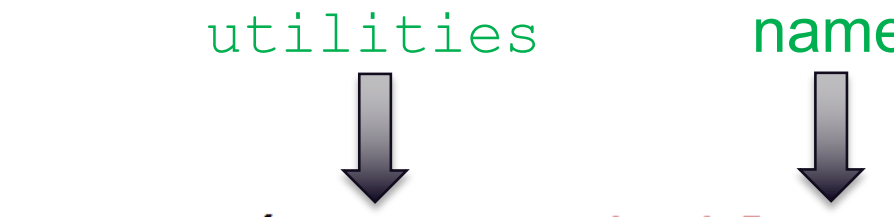

Test t(GROUP, "double_to_string", "fast");

check_to_disable_test(t, disabled_test_count);

if (t.is_enabled( ))

\{

double $x=-1.0$ e99;

std:: string expected = " $-1.000000 \mathrm{e}+99 "$;

std:: string actual = utils: :double_to_string $(x)$;

failed_test_count $+=$ t.check_equal(expected, actual);

\}

\}

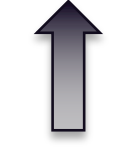

- returns 0 for PASS

- returns 1 for FAIL

J. Langr, "Modern C++ Programming with Test-Driven Development" (2013). 


\section{Compilation using the make utility}

- The source code is partitioned into logical units (classes, namespaces) that can be modified and tested (largely) independently.

- only changed source files need to be recompiled

- facilitates team collaboration and version control via repositories ( $g i t$ )

- All object code (except the small ma in) is assembled into a library file. - helps with possible future reuse in another application

- make

- compile the code (debug version) with the default compiler $(\mathrm{g}++)$

- make tests

- compile the unit-test suite

- make comp=intel opt=yes

- compile the code (optimized version) with a different compiler 


\section{Running the unit-test suite}

- / tests

- run all tests; only failed tests are printed

- / tests all verbose

- run all tests; the PASS or FAIL result is shown for all tests

- . tests <GROUP>

- run tests for the selected group, e.g., . / tests utilities

- . tests only <GROUP-test_name>

- run a specific test, e.g., . / tests only utilities-double_to_string

- Selected individual tests can be disabled. 


\section{Examples}

- The factorial function

- exact comparisons of integers ( int, also size_t, . . )

- Solution of the quadratic equation

- floating-point comparisons with a tolerance (double, also float )

- exact comparisons of strings ( string, also char)

- whole-object comparisons with a tolerance ( user-defined types ) 


\section{Procedure}

- Write the contract

- define the function's prototype (i.e., its inputs and outputs)

- Write unit tests, aim for full coverage of all logical paths

- compilation fails (i.e., testing the scope)

- Write code to return results outside the contract / unit tests

- test code runs, new unit tests fail (i.e., testing the tests)

- Write production code

- test code runs, all unit tests pass (i.e., newly added functionality works correctly and no previous code was broken) 


\section{Summary}

- Modularity and reusability of components

- large number of short routines and small files

- Design by contract

- specification of interfaces, up-to-date documentation

- Test-driven development with unit tests

- simple, but general, native unit-test framework

- Portability across platforms and compilers

- tested with Linux \& Mac platforms, GNU / Intel / Portland compilers

- Stateless kernels to aid parallelization \& threading

- data external to a class are passed via explicit argument lists instead of being shared via global variables 